Phương pháp số Sharpe-Lintner-Mossin : một ứng dụng trên số liệu thị trường chứng khoán Việt Nam 2000-2002

Vương Quân Hoàng

Nguyễn Quang Dong

Ngô Phương Chí

Tạp chí Nghiên cứu Kinh tế

Vol. 42, No. 12, Serial 295

Ngày 15 tháng 12 năm 2002 


\title{
Phương pháp số Sharpe-Lintner-Mossin: một ứng dụng trên số liệu thị trường chứng khoán Việt Nam 2000 - 2002
}

\author{
VƯƠNG QUÂN HOÀNG \\ NGUYỄN QUANG ĐÔNG \\ NGÔ PHƯƠNG CHÍ
}

\section{Giới thiệu}

Bài toán chúng ta sẽ xét dưới đây được thực hiện trên phương pháp số với số liệu thực tiễn. Thị trường chứng khoán Việt Nam (TTCKVN) đã tồn tại và phát triển được hơn 27 tháng. Trong suốt thời gian hoạt động đó, các nhà đầu tư luôn tìm kiếm các phương pháp tính toán hiệu quả để có thể điều chỉnh hành vi đầu tư, kể cả đầu cớ. Vì vậy, việc đưa ra một phương pháp số, dựa trên các kết quả lý thuyết có ý nghĩa thực tiễn rất cao, và không chỉ là mối quan tâm của người trực tiếp dầu tư. Trên thực tế, khắp nơi trên thế giới, các công ty tài chính, tư vấn và nhóm tính toán liên tục cải thiện các phương pháp số phục vụ ứng dụng trong đời sống kinh tế. Bài viết không nằm ngoài mục tiêu này, sẽ trực tiếp thực kiểm trên số liệu thống kê. Chúng tôi trân trọng cảm ơn Công ty tài chính Mezon (Mezfin) dã cung cấp số liệu từ cơ sở dũ liệu chính xác, khoa học và đầy đủ.

Một cách tóm tắt, phương pháp số Sharpe-Lintner-Mossin được hình thành từ việc mở rộng mô hình toán một thời kỳ của Markowitz. Các kết quả khoa học nền tảng được phát hiện từ việc kết hợp tài sản phi rủi ro (rủi ro bằng không), chính là trái phiếu kho bạc chính phủ, với các tài sản rủi ro, được trình bày trong ấn phẩm của các nhà khoa học W. Sharpe (1964), J. Lintner (1965) và J. Mossin (1966). Tuy nhiên, các nhà khoa học này đưa ra mô hình tổng quát, với số liệu toàn thị trường, vốn dĩ được tổ chức rất khoa học ở các nước phát triển. Điều này, chúng tôi không có được. Vì thế phương pháp số ứng dụng ở đây cho 5 cổ phiếu đầu tiên tồn tại trên TTCKVN, có chuỗi số liệu đủ dài để phản ánh hành vi lợi suất. Kết quả quan trọng nhất là tính ứng dụng cao, khả năng tính toán chính xác, góp phần phát triển nhận thức và phương pháp đầu tư.

\section{Phương pháp luận và số liệu}

\subsection{Phương pháp luận}

Trước tiên ta xem xét cơ sở khoa học của phương pháp tối ưu hóa đầu tư chứng khoán có rủi ro của $\mathrm{H}$. Markowitz như hệ tiền đề cho việc mở rộng mô hình số SharpeLintner-Mossin.

\section{Mô hìn Markowitz:}

Về bản chất là phần phát triển ứng dụng quan trọng của giải tích lồi, do Markowitz đưa ra lần đầu tiên năm 1952, cùng với một số phương pháp qui hoạch tuyến tính. Có thể nói đây là một trong những công trình kinh điển tính toán khoa học và có cơ sở vững chắc. Mô hình có dạng:

$$
\begin{aligned}
\operatorname{Min} \delta_{p}^{2}= & \mathrm{X}^{\prime} \mathrm{WX} \\
& \text { s.t. } \\
& X^{\prime} E=E_{p} \\
& X^{\prime} U=1
\end{aligned}
$$

Trong đó:

$$
\mathrm{E}=\left(\mathrm{E}_{1}, \mathrm{E}_{2}, \ldots, \mathrm{E}_{5}\right)^{\prime}
$$

$\mathrm{U}=(1,1, \ldots, 1)^{\prime} \quad$ và $\mathrm{X}=\left(\mathrm{X}_{1}, \mathrm{X}_{2}, \ldots, \mathrm{X}_{\mathrm{k}}\right)^{\prime}$ là véc - tơ tỉ trọng dầu tư trong danh mục tài sản tài chính. Việc giải bài toán này được trình bày tóm tắt dưới đây (độc giả có thể tham khảo V.Q.Hoàng \& N.T.Vinh (2001)

\footnotetext{
Vương Quân Hoàng, Mezfin Research; Nguyễn Quang Đông, Đại học kinh tế quốc dân; Ngô Phương Chí, Bộ Tài chính.
} 
về chi tiết của phương pháp nghiệm giải tích của Markowitz). Dưới đây sẽ mô tả tóm tắt phương pháp và kết quả.

Trước tiên hàm Lagrange của hàm mục tiêu được xác lập:

$\mathrm{L}\left(\mathrm{X}, \lambda_{1}, \lambda_{2},\right)=\mathrm{X}^{\prime} \Omega \mathrm{X}+2 \lambda_{1}\left(\mathrm{E}_{\mathrm{p}}-\mathrm{X}^{\prime} \mathrm{E}\right)+2 \lambda_{2}\left(1-\mathrm{X}^{\prime} \mathrm{U}\right)$

Rồi ta khai triển được hệ:

$$
\begin{aligned}
& \mathrm{X}=\lambda_{1} \Omega^{-1} \mathrm{E}+\lambda_{2} \Omega^{-1} \mathrm{U} \\
& \mathrm{E}^{\prime} \mathrm{X}=\mathrm{E}_{\mathrm{p}}=\lambda_{1} \mathrm{E}^{\prime} \Omega^{-1} \mathrm{E}+\lambda_{2} \mathrm{E}^{\prime} \Omega^{-1} \mathrm{U} \\
& \mathrm{U}^{\prime}=1=\lambda_{1} \mathrm{U}^{\prime} \Omega^{-1} \mathrm{E}+\lambda_{2} \mathrm{U}^{\prime} \Omega^{-1} \mathrm{U} \\
& \mathrm{a}=\mathrm{U}^{\prime} \Omega^{-1} \mathrm{E} \\
& \mathrm{b}=\mathrm{E}^{\prime} \Omega^{-1} \mathrm{E} \\
& \mathrm{c}=\mathrm{U}^{\prime} \Omega^{-1} \mathrm{U} \\
& \mathrm{d}=\mathrm{bc}-\mathrm{a}^{2}
\end{aligned}
$$

Trên thực tế ta đã có được phương trình bậc nhất sau:

$$
\begin{aligned}
& \lambda_{1} \mathrm{~b}+\lambda_{2} \mathrm{a}=\mathrm{E}_{\mathrm{p}} \\
& \lambda_{1} \mathrm{a}+\lambda_{2} \mathrm{c}=1
\end{aligned}
$$

Nghiệm thu được là: $\lambda_{1}=\frac{c E_{p}-a}{d}$;

$\lambda_{2}=\frac{b-c E_{p}}{d}$. véc - tơ $\mathrm{X}$ cần tìm có dạng giải tích:

$$
\mathrm{X}=\left(\frac{\mathrm{c}}{\mathrm{d}} \Omega^{-1} \mathrm{E}-\frac{\mathrm{a}}{\mathrm{d}} \Omega^{-1}\right) \mathrm{E}_{\mathrm{p}}+\left(\frac{\mathrm{b}}{\mathrm{d}} \Omega^{-1} \mathrm{U}-\frac{\mathrm{a}}{\mathrm{d}} \Omega^{-1} \mathrm{E}\right)
$$

Như vậy, ta có thể viết dạng nghiệm giải tích rút gọn của quá trình tối ưu hóa này:

$\mathrm{X}=\mathrm{G} E_{\mathrm{p}}+\mathrm{H}$

bằng cách đặt: $\mathrm{G}=\frac{\mathrm{c}}{\mathrm{d}} \Omega^{-1} \mathrm{E}-\frac{\mathrm{a}}{\mathrm{d}} \Omega^{-1} \mathrm{U}$, và $\mathrm{H}=\frac{\mathrm{b}}{\mathrm{d}} \Omega^{-1} \mathrm{U}-\frac{\mathrm{a}}{\mathrm{d}} \Omega^{-1} \mathrm{E}$

Tiếp theo, thay trở lại mô hình ban đầu, và đặt:

$$
\mathrm{k}_{0}=\frac{\mathrm{b}}{\mathrm{d}} ; \mathrm{k}_{1}=\frac{2 \mathrm{a}}{\mathrm{d}} ; \mathrm{k}_{2}=\frac{\mathrm{c}}{\mathrm{d}}
$$

phương trình toàn phương của phương sai theo kỳ vọng lợi suất có dạng:

$$
\delta_{\mathrm{p}}^{2}=\mathrm{k}_{2} \mathrm{E}_{\mathrm{p}}^{2}+\mathrm{k}_{1} \mathrm{E}_{\mathrm{p}}+\mathrm{k}_{0}
$$

\section{Mô hình Sharpe-Lintner-Mossin:}

Trên thực tế, việc loại tài sản phi rủi ro khỏi mô hình tối ưu hóa không cần thiết. Ngược lại, đưa thêm tài sản phi rủi ro, ví dụ trái phiếu kho bạc ngắn hạn như sẽ xét trong bài toán ứng dụng, hoàn toàn có thể góp phần tối ưu hóa hành vi đầu tư. Mô hình mở rộng Sharpe-Lintner-Mossin đề cập đến vấn đề này. ở đây, chúng ta không xét các vấn đề rộng hơn như định giá tài sản vốn $(\mathrm{CAPM})$, mà tập trung vào hành vi khi tài sản phi rủi ro được bổ sung. Về mặt hình học, ta có:

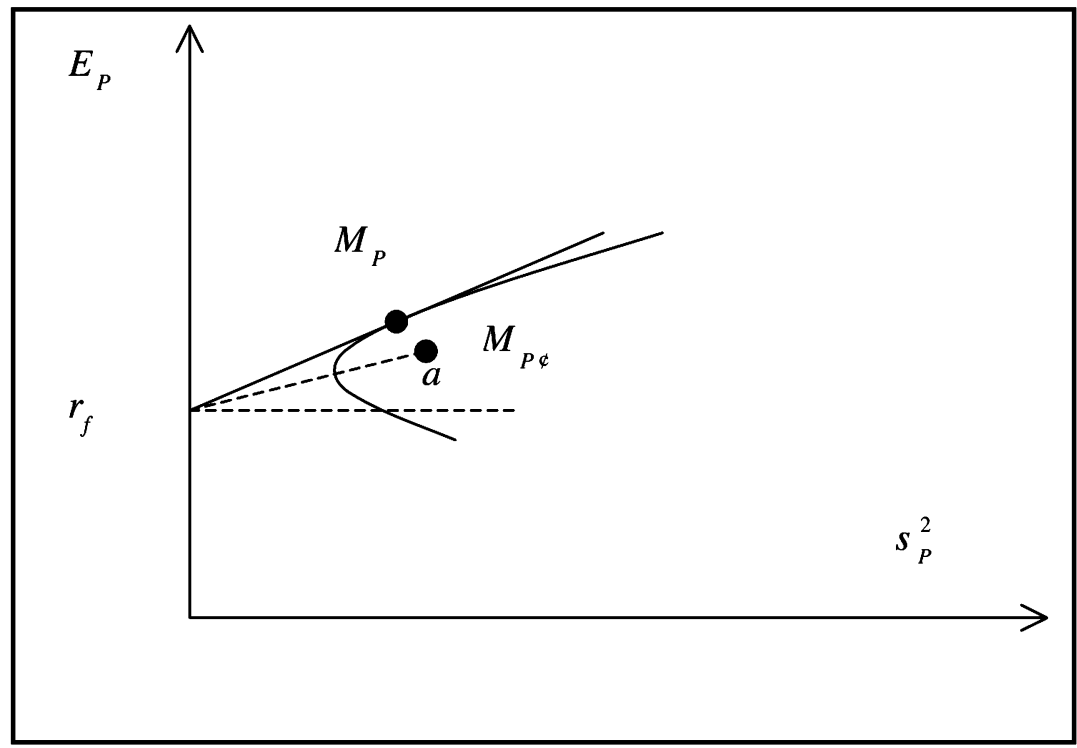

Điều chúng ta quan tâm, về định tính, là tiếp tuyến kẻ từ $\left(r_{f}, 0\right)$ qua $\mathrm{M}_{\mathrm{p}}$ là đường khả thi đầu tư. Ta gọi $\mathrm{M}_{\mathrm{p}}$ là danh mục thị trường, trong trường hợp danh mục này có tính chất tương đối đại diện cho nhóm đông các cổ phiếu, chẳng hạn mô phỏng hành vi 
của một chỉ số chứng khoán.

Ta cần điều chỉnh mô hình như sau. TTCKVN không cho phép bán khống do đó, điều quan trọng bậc nhất của mô hình tối ưu là ràng buộc bổ sung:

$\mathrm{X}_{\mathrm{i}} \geq 0 ; \forall_{\mathrm{i}}$

Trong trường hợp này, nghiệm giải tích hầu như không khả thi, và phương pháp số thông qua lập trình. Xét một trường hợp lý thuyết như sau. Giả sử nghiệm của phương pháp số được biểu diễn qua chấm thứ hai (nằm dưới tiếp điểm danh mục thị trường, ở hình vẽ trên), như vậy ta có thể tìm được một tập hợp các điểm khác, cùng thoả mãn hệ hàm tối ưu có bổ sung ràng buộc không âm. Vậy cần có dấu hiệu cho quá trình lựa chọn các điểm danh mục sao cho kết quả tối ưu. Đây là trái tim lý thuyết của SharpeLintner-Mossin được điều chỉnh trong trường hợp của chúng ta.

\begin{tabular}{|c|c|c|c|c|c|c|c|}
\hline \multirow{5}{*}{$\mathrm{E}=$} & 0.001875 & & 0.005848 & 0.002976 & 0.002760 & 0.003775 & 0.003713 \\
\hline & 0.005732 & Và & 0.002976 & 0.003058 & 0.002177 & 0.002933 & 0.002766 \\
\hline & 0.007203 & $\Omega=$ & 0.002760 & 0.002177 & 0.005765 & 0.003088 & 0.002610 \\
\hline & 0.009474 & & 0.003775 & 0.002933 & 0.003088 & 0.004797 & 0.003879 \\
\hline & 0.002234 & & 0.003713 & 0.002766 & 0.002610 & 0.003879 & 0.005067 \\
\hline
\end{tabular}

Các số liệu này vừa là điều kiện cần, và điều kiện đủ cho tiến hành các nghiên cứu trên nghiệm số dưới đây.
Chúng tôi đề xuất việc đưa ra dấu hiệu lựa chọn lợi ích lớn nhất của đầu tư danh mục. Kẻ một đường nối $\left(\mathrm{r}_{\mathrm{f}}, \mathrm{M}_{\mathrm{p}}{ }^{\prime}\right)$ và kẻ một đường song song với trục hoành. Ta thu được $\Varangle \alpha$, và việc so sánh sẽ theo: $\max (\tan (\alpha))$, tạm gọi là dấu hiệu ưu tiên. Bản chất của việc sử dụng hệ số ưu tiên theo đề xuất của chúng tôi chỉ đơn giản là lựa chọn hệ số góc lớn nhất, nhằm tối đa hóa hành vi hàm hiệu dụng.

\subsection{Sốliệu}

Dưới đây là trình bày vắn tắt về dữ liệu của mô hình. Dựa trên cơ sở dữ liệu của Công ty Tài chính Mezon (Mezfin), chúng tôi đưa ra một danh mục 5 cổ phiếu, bao gồm REE, SAM, HAP, TMS, và LAF. Gói số liệu kết thúc ở phiên 386. Theo đó các phần tử đầu vào là véc-tơ lợi suất trung bình tuần và ma trận hiệp phương sai (các phần tử trên đường chéo là phương sai):
3. Phương pháp số, kết quả và so sánh

Trong trường hợp tìm nghiệm giải tích, ta thu được kết quả thực nghiệm như sau:

$$
\Omega^{-1}=\left|\begin{array}{rrrrr}
416.7036761 & -183.0819705 & -19.21914346 & -119.4704812 & -104.1056795 \\
-183.0819705 & 938.6783828 & -52.06799327 & -294.5225463 & -125 .-170026 \\
-19.21914346 & -52.06799327 & 270.3801565 & -128.1847935 & 1.348816626 \\
-119.4704812 & -294.5225463 & -128.1847935 & 816.1567382 & -310.4656741 \\
-104.1056795 & -125.9170026 & 1.348816626 & -310.4656741 & 579.4082482
\end{array}\right|
$$

$$
\mathrm{G}=\left|\begin{array}{c}
-52.48575288 \\
2.362277663 \\
0.475916906 \\
134.0502938 \\
-84.40273552
\end{array}\right| \quad \text { Và }=\left|\begin{array}{c}
0.254266592 \\
0.796307193 \\
0.203932406 \\
-0.820617167 \\
0.566110976
\end{array}\right|
$$


và các hệ số của phương trình bậc hai biểu diễn biên hiệu dụng là:

$$
\begin{aligned}
& \mathrm{k}_{0}=0.003727911 \\
& \mathrm{k}_{1}=-0.325750618 \\
& \mathrm{k}_{2}=30.47855143
\end{aligned}
$$

Như vậy, phương trình quan hệ có dạng: $\delta_{\mathrm{p}}^{2}=30.478551 \mathrm{E}_{\mathrm{p}}^{2}-0.3257506 \mathrm{E}_{\mathrm{p}}+0.0037279$
(So sánh với V.Q.Hoàng và N.T.Vinh (2001), kết quả này đã dịch chuyển đáng kể, do hai quá trình động học khác nhau của cả lợi suất lẫn phương sai, tương ứng với quan điểm méo phương sai trên mẫu nhỏ). Đồ thị nghiệm giải tích trường hợp không ràng buộc.

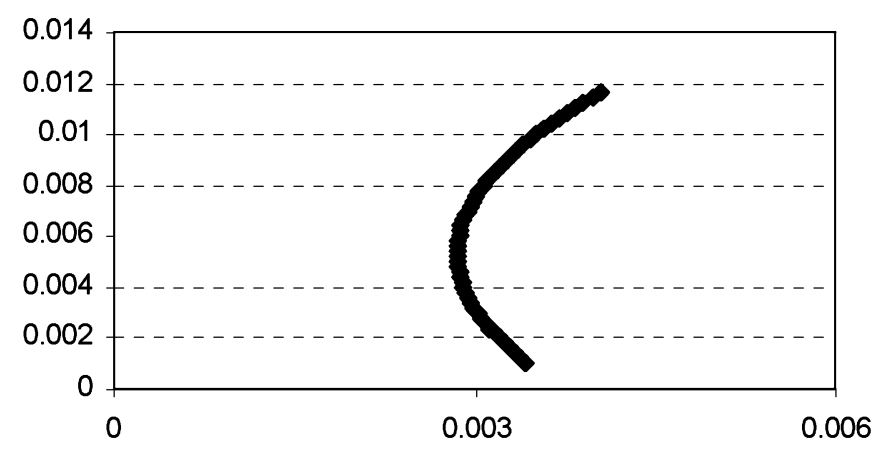

Tuy nhiên, phần quan trọng nhất là tôi ưu hóa phương pháp số. Chúng tôi tiến hành công việc dựa trên thuật toán tôi ưu hóa phi tuyến, môi trường Mathematica của Culioli (1994). Việc tìm kiếm nghiệm số hoàn toàn đơn giản. Sử dụng thuật toán chung nói trên, chúng tôi viết lại hàm mục tiêu (toàn phương) cho phương trình trung bình-phương sai, và truyền tham số vào cho solver tìm kiếm cực tiểu cục bộ. Rõ ràng, kết quả sẽ là các điểm rời rạc, khi ta thay đổi tham số hàm mục tiêu, cư thể là phương sai hoặc lợi suất kỳ vọng. Đồ thị nghiệm phương pháp số với ràng buộc thị trường dưới đây là các điểm toạ độ tìm được qua việc tôi hóa theo phương pháp trên:

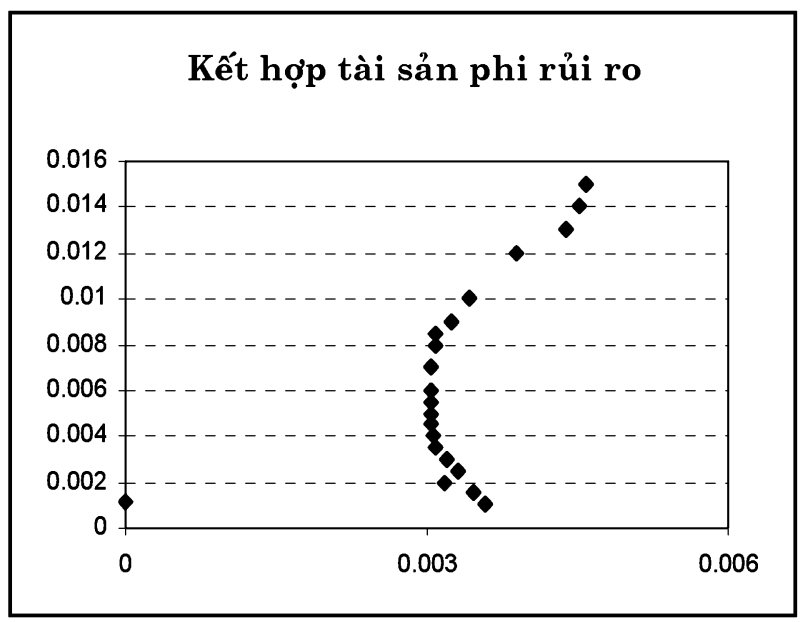

Đồ thị ở hình trên cho thấy các điểm rời rạc, và biến động khá kỳ dị, mặc dù ta đã lấy các điểm rất gần nhau về mặt hình học. Lưu ý, điểm nằm trên trục tung chính là số liệu thực của (rf,o). Nếu so sánh với dạng đồ thị nghiệm giải tích phía trên, thì không thể so sánh về tính đẹp của kết quả. Tuy vậy, tính xác thực của nghiệm trên môi trường ràng buộc được công nhận. Trong những trường hợp tôi ưu hóa nhiều phương trình ràng buộc như trên, việc xử lý lựa chọ theo dấu hiệu là cần thiết. Do vậy, dưới đây là đồ thị của các hệ số góc được lựa chọn.

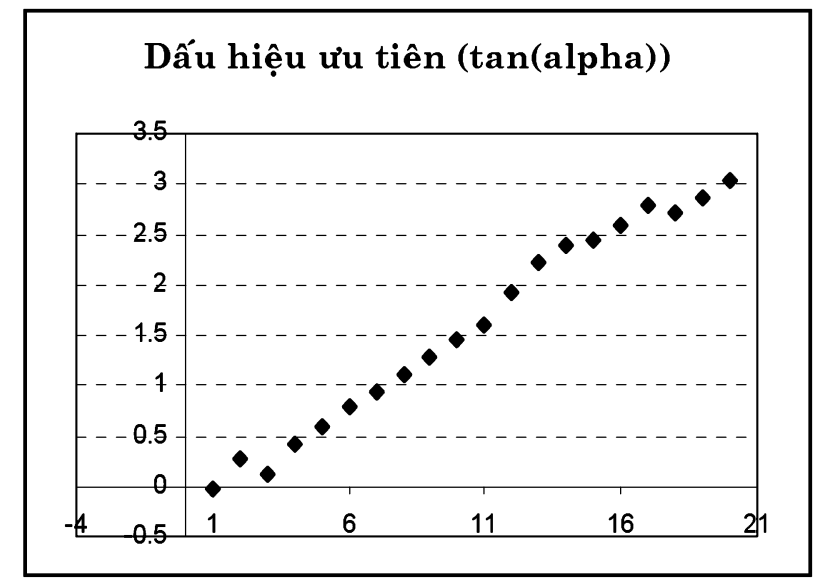

Cụ thể về tính toán theo phương pháp số, ta thu được bảng kết quả dưới đây. Bảng cho thấy các giá trị của dấu hiệu thay đổi thất thường. Do đó, quá trình lựa chọn là do người 
sử dụng. Ví dụ, khi xác định rõ được khoảng mục tiêu lợi suất, dấu hiệu sẽ được xem xét cả về giá trị tuyệt đối cũng như so sánh tốc độ tăng khi các tham số khác thay đổi.

\begin{tabular}{rrr}
\hline Phương sai & $\begin{array}{c}\text { Lợi suất kỳ } \\
\text { vọng }\end{array}$ & \multicolumn{1}{c}{$\begin{array}{c}\text { Dấu hiệu ưu } \\
\text { tiên }\end{array}$} \\
\hline 0.00357311 & 0.001 & -0.0322925 \\
0.00318978 & 0.002 & 0.277328 \\
0.00346132 & 0.0015 & 0.1111181 \\
0.00331214 & 0.0025 & 0.4180425 \\
0.00320129 & 0.003 & 0.588705 \\
0.00308928 & 0.0035 & 0.7719 \\
0.00306815 & 0.004 & 0.9401807 \\
0.00305183 & 0.0045 & 1.1090445 \\
0.00304204 & 0.005 & 1.2769771 \\
0.00304628 & 0.0055 & 1.4393343 \\
0.00304736 & 0.006 & 1.6029007 \\
0.00305384 & 0.007 & 1.926956 \\
0.00309585 & 0.008 & 2.2238207 \\
0.00309941 & 0.0085 & 2.3825875 \\
0.00324755 & 0.009 & 2.4278657 \\
0.00342097 & 0.010 & 2.5971042 \\
0.00389795 & 0.012 & 2.7923948 \\
0.00438380 & 0.013 & 2.7110305 \\
0.00451748 & 0.014 & 2.8521688 \\
0.00458886 & 0.015 & 3.0257222 \\
\hline & & \\
& & 0.356
\end{tabular}

Các điểm xét lân cận sẽ giúp ta có tương quan so sánh. Lấy ví dụ, với nghiệm số tại điểm phương sai bằng 0.00438380 , và lợi suất kỳ vọng là 0.013 , thì hệ số lại giảm đi. Như vậy, không thể chọn phương án này, mà cân nhắc giá trị đứng trước hay sau, theo mức rủi ro thích hợp cho lựa chọn.

Như vậy, trong bài viết này chúng ta đã xem xét một ứng dụng quan trọng của bài toán tối ưu hóa danh mục đầu tư, trong điều kiện mở rộng có ràng buộc thị trường. Phương pháp số cho kết quả, và ta cũng phát triển dấu hiệu so sánh cho việc lựa chọn. Dâu hiệu ưu tiên đã xét là đề xuất riêng của chúng tôi và cần thiết, vì trong trường hợp cụ thể này, bản thân danh mục không phải là danh mục thị trường, mà chỉ gói gọn trong nhóm 5 cổ phiếu xem xét. Tính ứng dụng của bài toán khá cao, và có thể là dấu hiệu chung cho các nhà phân tích trường phái trung bình-phương sai./.

\footnotetext{
TÀI LIÊU THAM KHẢO

- Culioli, J-C. (1996) Optimization with Mathematica, in Ed. Varian, H. Computational Economics and Finance. Springer-Velag, TELOS. - V.Q.Hoàng, và N.T. Vinh (2001) Tối ưu hãa portfolio Markowitz-Sharpe: Thị trường chứng khoán Việt Nam sau 8 tháng hoạt động, TC Nghiên cứu kinh tế, 41, 5, 19-28.

V.Q.Hoàng, (2002) Empirical evidence of conditional heteroskedasticty in Vietnam's stock returns time series, Social Science Research Network, Oct 2002.

- Markowitz, Harry M. (1952) Portfolio selection, J. of Fin. 7, 77-91

- Mossin, Jan (1966) Equilibrium in a capital asset market, Econometrica 34, 768-783.

- Lintner, John (1965) The valuation of risk assets and the selection of risky investment in stock portfolio and capital budgets, Rev. Econ. \& Stat. 47, 13-37.

- Samuelson, Paul (1938) The empirical implication of utility analysis, Econometrica 6, 344-356.

- Sharpe, William F. (1964) Capital asset prices: a theory of market equilibrium under conditions of risk, J. of Fin. 19, 425-442.

Sharpe, William F. (1970) The portfolio theory and capital markets. McGraw-Hill, CA.

- Sharpe, William F. (1991) Capital asset prices with and without negative holdings, J. of Fin. 46, 489-509.
} 